\title{
Current applications of the microbiome engineering and its future: A brief review
}

\author{
Zahra Malayejerdi¹, Omid Pouresmaeil1,** \\ ${ }^{1}$ Department of Medical Laboratory Sciences, Varastegan Institute for Medical Sciences, Mashhad, Iran
}

\begin{abstract}
In the human body there are many microorganisms with a variable genetic content. These microorganisms play an important role in the metabolism, homeostasis, immune system and generally human health. Over the millions of years, different microorganisms adapted to each other, and different environmental communities formed on Earth. Microbial communities, known as microbiome, could exist in living or non-living environments, such as human body and plants, as well as in soil, oceans, and air. The main purpose of microbiome engineering is mostly human microbiome and is now used in the treatment of diseases such as Clostridium difficile infection, inflammatory bowel disease, obesity, etc. The research data in this thesis were collected from the main medical article sources including Web of science, Google scholar, PubMed and Scopus. Articles on microbiome which published during 20102019 were reviewed. The widespread impacts of the microbiome on the ecosystems and the increased attention to microbiome recognition are factors contributing to the creation of microbiome engineering science, and recent advances in genome sequencing and metagenomic science have made microbiome analysis apart from cultivation process. Microbiome engineering has advantages and disadvantages. So, according to the positive aspects and efforts to increase applications, this science could lead to advances in microbial engineering, and have positive effects on human health. Although microbiome engineering is a new field, there has been lots of progressions in recent years that can be an important strategy for improving human health by microbial manipulation leading to the changing of microbial population.
\end{abstract}

Keywords: Microbiome, Human Gut Microbiome, Microbiome engineering, Microbiota

\section{Introduction}

There are many microorganisms in the human body with a wide variety of genetic content. These microbes play an important role in metabolism, homeostasis, immune system, and human health. However, most microbes cannot be cultured in a laboratory environment except for a limited number of bacteria [1]. The mankind has almost the same genetic patterns; just a little difference in DNA lead to phenotypic diversity among the human population, but the human microbiome metagenomic, which is, the whole genome of microbes living in the body, is completely variable and unique. Therefore, understanding the diversity of healthy microbiome is a major challenge in microbiome research and dates back to the 1960 s and continues through the Human Microbiome Project [2, 3]. The Human Microbiome Project is a project launched by the National Institute of Health (NIH) to identify and analysis of all types of microorganisms in the human and the term

\footnotetext{
${ }^{*}$ Corresponding author:

Omid Pouresmaeil, MSc

Department of Medical Laboratory Sciences,

Varastegan Institute for Medical Sciences, Mashhad, Iran

Tel/Fax: +989153752499

Email: omidpb71@gmail.com

http://orcid.org/0000-0002-0548-2294
}

Received: August, 14, 2020

Accepted: September, 18, 2020
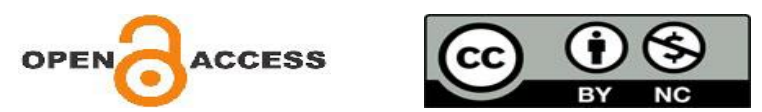
"Microbiome" was used first time by Joshua Lederberg in 2001 [4, 5]. Through evolution; over millions of years, different organisms have adapted to each other, and different environmental communities have formed on Earth. Microbial communities, known as microbiome, can exist in living or non-living environments such as the human body, animals, and plants, as well as in soil, oceans, and air. The microorganisms that make up microbiome are actively compatible with the hosts and interactions affect on characteristics of the ecosystems in which they live. It can also have a wide range of effects on physiology (host or environmental conditions). Therefore, the widespread impact of the microbiome on the ecosystem and increased attention to microbiome recognition are factors affecting the development of microbial engineering. Recent advancements in the sequencing of genome and metagenomic science have made it possible to analyze microbiome independent of culture techniques [6]. The term "Microbiome" refers to all microorganisms [including Archaea, bacteria, eukaryotes, and viruses], genomes and their environmental conditions, and the term "bio" it is an environmental community it has a special climate, plants and animals where they live. In other words, a microbiome is a combination of metagenomic, metabonomic, metatranscriptomic, and metaproteomic that is described along with comprehensive environmental or clinical information. Metagenomic, metabonomic, metatranscriptomic and metaproteomic are sciences that have been formed around the axis of microbiome engineering. Microorganisms in the environment are called microbiota. The term microbiota was first coined by Lederberg and McCray; human microbiota is made up of many microorganisms, including microbes on the surface of the skin, genital tract, and gastrointestinal tract [6]. The microbiome is a complex term that is sometimes mistakenly used instead of microbiota but, they have different meanings. The term metagenomic is used to describe the sequence of microbial DNA; nowadays, the 16SrRNA marker is used. More than 1,000 intestinal bacterial species have already been discovered; Bacteroides have previously been the most common intestinal bacteria, classified into five genera: Alistipes, Prevotella, Paraprevotella, Parabacteroides, and Odoribacter. Metataxonomics is the science that describes the whole microbiota and their phylogenetic relationships through the analysis of their genome. The term microblome is an analytical method in which the metabolic profile of a microbiota is estimated and described. Metatranscriptomics analyzes transcriptional mRNAs, and metaproteomics addresses the characteristics of all microbiota proteins, first developed by Rodrigue-vaiera [7, 8]. Microbiome engineering it tries to shape the microbiota to change ecosystems, the characteristics of ecosystems are determined by the main microbiome. The unique feature of each microbiome is the result of the interaction of microorganisms and host communities, as well as the response to metabolites produced by microbial communities. Therefore, Disruption of microbiomes can cause extensive changes in the host and the environment, the main goal of microbiome engineering is the more human microbiome and today it is used in the treatment of diseases. The human microbiome project to a large extent advanced metagenomic microbial properties in the human body, it describes the intestines, mouth, skin, lungs, nose, and urinary system. The information obtained from the Human Microbiome Project has made microbial engineering dramatically provide solutions to restore the microbial balance with therapeutic functions $[2,6]$.

\section{Methods}

In the present study, related articles were collected from main sources such as Web of Science, Google scholar, PubMed, and Scopus. The selected articles were published between 2010 and 2019, due to the remarkable progress that microbiome engineering has made during this period. Microbiome engineering has reached its peak in the coming years and has a very bright future ahead.

\section{Discussion}

\subsection{Fecal microbiota transplantation}

Jang Lee et al. after receiving healthy feces from a healthy donor, prepared a suspension and injected it into the gastrointestinal tract of the recipient. They reported fecal microbiota transplantation (FMT) or fecal transplantation was performed to heeling Clostridium difficile infection and restoring the gut microbiome; It is also reported to be effective in treating Inflammatory Bowel Disease (IBD), diarrhea, food poisoning, or other gastrointestinal diseases such as rheumatoid arthritis, autism, cancer, weight loss and even person's mood and behavior [6, 9-11]. 


\subsection{Reduce muscle pain and probiotic}

Scheiman et al. by study on the gut microbiome reported that Veillonella atypica has an important role to convert the acetic acid to propionate [12]. Lactic acid is caused by prolonged exercise and causes muscle pain, while propionate is used by cells to improve exercise ability. The study has shown that $V$. atypica can increase running time in marathon runners [12]. Yatsonko et al. noted the effects of the microbiome in infants. Over time, as a result of contact with the environment, more bacteria enter the baby's body, and the organisms entering to the body in the first days of life will most likely remain for the rest of life. Depending on the type of delivery and nutrition condition, the microbiome may be different. The study noted that the type of delivery affects on baby's immune system. Natural delivery leads to stronger immune system development in babies [2]. Jernberg et al. assessed the role of antibiotics and probiotics in association with human microbiome. This study states that antibiotics are the most effective agents on intestinal homeostasis. Probiotics are usually a combination of bacteria that produce lactic acid, such as lactobacilli, Bifidobacteria, which are important for an appropriate health level [1].

\subsection{Stress and depression}

Nofeld et al. experimented on female mice to show the absence of normal microbiota could lead to increased anxiety behaviors. Bilateral communication between the gastrointestinal tract and the brain is important for maintaining intestinal homeostasis. This connection is made by the central nervous system. Disruption of these systems can lead to changes in response to stress and general behaviors. The results declared that normal microbiota reduces anxiety [13, 14]. Serotonin is known as a neurotransmitter that affects the feeling of happiness and regulates mood. In fact, more than $90 \%$ of the serotonin produced is in the gut [15]. O'mahoni et al. declared a relation between the intestinal microbiome and the synthesis of serotonin and its effect on depression. They noted that the intestinal microbiome could be a factor in regulating intestinal serotonin production. Many intestinal bacteria can produce small molecules such as serotonin, epinephrine, and norepinephrine. Clostridium sporogenes can produce tryptophan, stimulating the production of serotonin. Escherichia coli and other bacteria such as enterococci can produce serotonin and epinephrine. These hormones regulate many of the functions of the nervous system in the brain by producing other hormones affecting anxiety and stress [3, 15-17]. The study mentioned intestinal microbial disorders, impaired intestinal serotonin synthesis, leading to depression [3, 15-17]. Microbiome engineering with a specific metabolic network makes it possible to produce specific products; so, the metabolic activities of each microbiome leads to a special type of biological product with unique properties.

\subsection{Future and limitations of microbiome engineering}

Microbiome engineering can produce or detect intelligent microbes that have new and unique properties, which can be used to treat some diseases [6]. For example in experiments FMT have been used to treat many diseases, including $C$. difficile, to restore the gut microbiome [6]. Additionally, some bacteria can be generated which selectively could potentially use to eradicate strains of dangerous microbiota in the body. For example $E$. coli has been engineered to interrupt signaling between microbes as a means of altering the microbiota [18]. Despite extensive research on the human microbiome, understanding of the microbiota ecosystem is still limited and the dynamics of the microbiome and the interactions between microbes are not well understood $[4,6]$.

\section{Conclusions}

Although microbiome engineering passes several developments in recent years, it still needs lots of researches and studies. Studies on human microbiomes which have been performed using metagenomic, highlight the undeniable functional role of microbiome in the body, and it provides new clinical therapeutic methods related to the microbiome. Microbiome engineering is an important strategy for improving human [6].

\section{Author Contributions}

All authors contributed equally to this manuscript and approved the final version of manuscripts.

\section{Conflict of Interests}

Authors declare there is no conflict of interest. 


\section{Ethical declarations \\ Not applicable.}

\section{Financial Support}

None.

\section{References}

1. Kim BS, Jeon YS, Chun J. Current status and future promise of the human microbiome. Pediatr Gastroenterol Hepatol Nutr. 2013; 16(2):71-9.

2. Lloyd-Price J, Abu-Ali G, Huttenhower C. The healthy human microbiome. Genome Med. 2016; 8(1):51.

3. Bäckhed F, Fraser CM, Ringel Y, Sanders ME, Sartor RB, Sherman PM, et al. Defining a healthy human gut microbiome: current concepts, future directions, and clinical applications. Cell Host Microbe. 2012; 12(5):611-22.

4. Ursell LK, Metcalf JL, Parfrey LW, Knight R. Defining the human microbiome. Nutr Rev. 2012; 70 Suppl 1(Suppl 1):S38-44. 5. Prescott SL. History of medicine: Origin of the term microbiome and why it matters. Hum Microbiome J. 2017; 4:24-5.

6. Foo JL, Ling H, Lee YS, Chang MW. Microbiome engineering: Current applications and its future. Biotechnol J. 2017; 12(3).

7. Marchesi JR, Ravel J. The vocabulary of microbiome research: a proposal. Microbiome. 2015; 3(1):31.

8. Phillips CD, Phelan G, Dowd SE, McDonough MM, Ferguson AW, Delton Hanson J, et al. Microbiome analysis among bats describes influences of host phylogeny, life history, physiology and geography. Mol Ecol. 2012; 21(11):2617-27.

9. Jung Lee W, Lattimer LD, Stephen S, Borum ML, Doman DB. Fecal Microbiota Transplantation: A Review of Emerging Indications Beyond Relapsing Clostridium difficile Toxin Colitis. Gastroenterol Hepatol (NY). 2015; 11(1):24-32.

10. Culligan EP, Sleator RD. Advances in the Microbiome: Applications to Clostridium difficile Infection. J Clin Med. 2016; 5(9).

11. Hansen JJ, Sartor RB. Therapeutic Manipulation of the Microbiome in IBD: Current Results and Future Approaches. Curr Treat Options Gastroenterol. 2015; 13(1):105-20.

12. Scheiman J, Luber JM, Chavkin TA, MacDonald T, Tung A, Pham L-D, et al. Meta-omics analysis of elite athletes identifies a performance-enhancing microbe that functions via lactate metabolism. Nat Med. 2019; 25(7):1104-9.

13. Foster JA, McVey Neufeld KA. Gut-brain axis: how the microbiome influences anxiety and depression. Trends Neurosci. 2013; 36(5):305-12.

14. Eming SA, Martin P, Tomic-Canic M. Wound repair and regeneration: mechanisms, signaling, and translation. Sci Transl Med. 2014; 6(265):265sr6.

15. O'Mahony SM, Clarke G, Borre YE, Dinan TG, Cryan JF. Serotonin, tryptophan metabolism and the brain-gut-microbiome axis. Behav Brain Res. 2015; 277:32-48.

16. Dinan TG, Stilling RM, Stanton C, Cryan JF. Collective unconscious: how gut microbes shape human behavior. J Psychiatr Res. 2015; 63:1-9.

17. Babakhani S, Hosseini F. Gut Microbiota: An Effective Factor in the Human Brain and Behavior. Shefaye Khatam. 2019; 7(1):106118.
18. Hwang IY, Koh E, Kim HR, Yew WS, Chang MW. Reprogrammable microbial cell-based therapeutics against antibiotic-resistant bacteria. Drug Resist Updat. 2016; 27:59-71. 